Www.sciencedirect.com

Journal of Statistical Planning and Inference 137 (2007) 2405-2412

www.elsevier.com/locate/jspi

0378-3758

(C)2006 Elsevier B.V. All rights reserved.

Doi:10.1016/j.jspi.2006.09.021

\title{
Generalized model for IGC growth in aluminum alloys
}

\author{
Shang Zhao ${ }^{a}$, Douglas A. Wolfe ${ }^{a, *}$, Tsai-Shang Huang ${ }^{b}$, Gerald S. Frankel ${ }^{b}$ \\ ${ }^{a}$ Department of Statistics, Ohio State University, Columbus, OH 43210, USA ${ }^{b}$ Department of \\ Materials Science and Engineering, Ohio State University, Columbus, OH 43210, USA
}

\begin{abstract}
A generalized brick wall model is developed to describe intergranular corrosion in Equi-axedAA7178-T6 and Wingskin AA7178-T6 aluminum alloys. The intergranular corrosion rate is highly related to grain size and shape. High strength aluminum alloys are often elongated and anisotropic, with the fastest nominal IGC growth rate in the longitudinal direction (L) or long transverse direction (T) and the slowest in the short transverse direction (S). We propose a three-way intersection model and use it to simulate the corrosion kinetics for each direction. With a proper combination of model parameters, the generalized IGC model provides a good fit to experimental data developed by the foil penetration technique.
\end{abstract}

\section{Introduction}

Aluminum alloys are widely used in aerospace applications because of their high strength and low weight. However, they are very susceptible to localized corrosion, which includes intergranular corrosion (IGC), pitting corrosion, crevice corrosion and stress corrosion cracking. Localized corrosion could result in structure failure of an aluminum alloy if it is allowed to reach critical levels. The microstructure of alloys is made up of grains, separated by grain boundaries. IGC is localized attack along the grain boundaries, or adjacent to grain boundaries (Guillaumin and Mankowski, 1999), while the bulk of the grains remains largely unaffected. This form of corrosion can be very dangerous because the attack can proceed undetected through the material and may deteriorate the mechanical properties of the metal and cause fracture without visible signs of corrosion. In addition, the speed of this IGC attack is faster than other types of localized corrosion. Thus, among all the forms of localized corrosion in aluminum alloys, IGC is of special interest, so that study of the corrosion growth kinetics of IGC is critical.

One factor that determines the resistance to IGC is alloy microstructure, including grain size and shape (Robinson and Jackson, 1999). High strength aluminum alloys are often elongated and anisotropic, with grains elongated in the longitudinal direction (L) or the long transverse direction (T) and short in the short transverse direction (S) (Liu et al., 2004). Huang and Frankel (2004) observed that corrosion is also anisotropic in such aluminum alloys. It was reported that the IGC kinetics in a rolled AA2024-T3 plate have different orientations relative to the rolling direction, with the fastest nominal IGC growth rate in the longitudinal direction (L) or long transverse direction (T) and the slowest in the short transverse direction (S). This is due to the straight IGC path along either the $\mathrm{L}$ or $\mathrm{T}$ directions and the circuitous path in the $\mathrm{S}$ direction (see Fig. 1). 

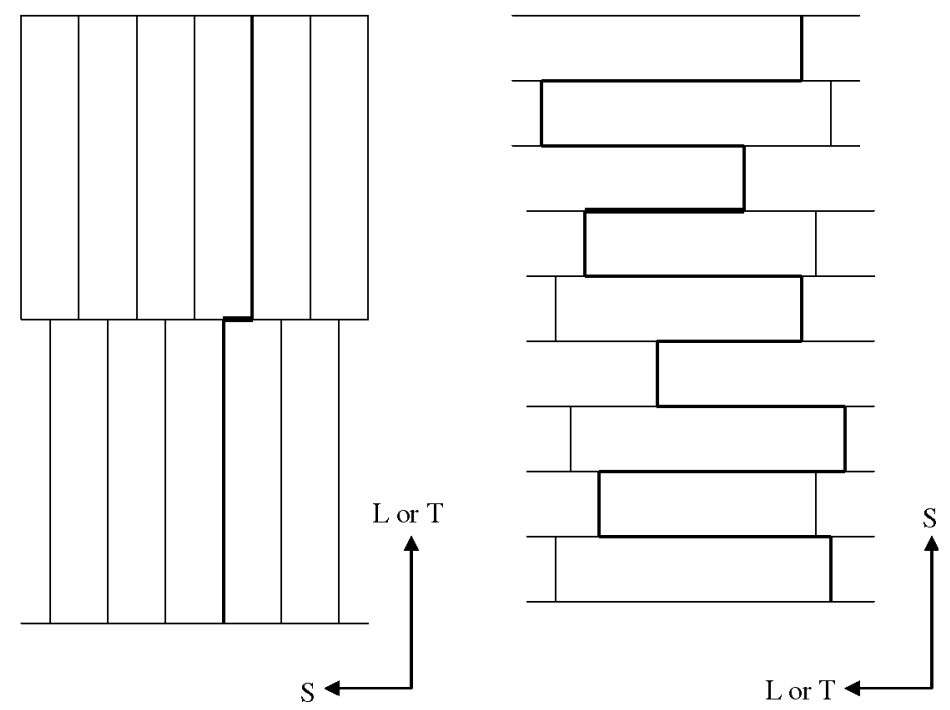

Fig. 1. The minimum IGC path in $\mathrm{L}$ or $\mathrm{T}$ direction, and in $\mathrm{S}$ direction.

Ruan et al. (2004a,b) proposed a brick wall model to describe the relationship between the microstructure and the IGC growth rate of AA2024-T3. However, the brick wall model was based on some simplifying assumptions. First, since the longitudinal and long transverse directions are much longer than the short transverse direction, the problem was simplified to consider only the S direction. However, the corrosion growth kinetics is different in S-L and ST sections. Moreover, in the Ruan et al. studies, when the corrosion path met an intersection, it was assumed to be a four-way intersection in the simulation, even though by nature of the aluminum alloy, three-way intersections are more common. Based on these considerations of the corrosion behavior, a more realistic modified brick wall model is presented in this paper.

In our experiment, we use the foil penetration technique to measure the corrosion rate in different orientations for two types of material: Equi-axed AA7178-T6 and Wingskin AA7178T6. Using this technique the penetration time for the fastest-growing IGC is measured for different sample thicknesses and in various sample orientations for these two aluminum alloys. The kinetics was determined by the penetration time and the depth of the corrosion. We will use simulations with the modified brick wall model to study the relationship between the corrosion rate and the alloy microstructure.

\section{Model and simulation}

\subsection{Model}

Modeling the relationship between the corrosion rate and the alloy microstructure must take into account two considerations. First, aluminum alloys are composed of grains. The anisotropy is due to different grain sizes in the three directions, so studying the micro-structure can be simplified to studying of the grain sizes of the different orientations. Second, it is reasonable to consider the local intergranular growth speed to be the same everywhere. Therefore, the fastest corrosion rate, as measured in the experiment, corresponds to the minimum IGC path length. Hence modeling the relationship between the corrosion rate and the alloy's microstructure is equivalent to modeling the relationship between the shortest corrosion path and grain sizes in the three directions. 
From the study by Huang and Frankel (2004), the grain sizes in all directions have distributions that are skewed to the right, so that gamma distributions are appropriate for modeling the grain size distributions for all three directions. We use the method of moments to estimate the parameters of these gamma distributions. In the experiment, we obtain the sample means, $M$, and sample standard deviations, $S$, of the grain sizes for each of the three directions. Method of moment estimates of the gamma distribution parameters $\alpha$ and $\beta$ can then be calculated from the following two equations:

$$
\alpha \beta=M
$$

and

$$
\alpha \beta^{2}=S^{2} .
$$

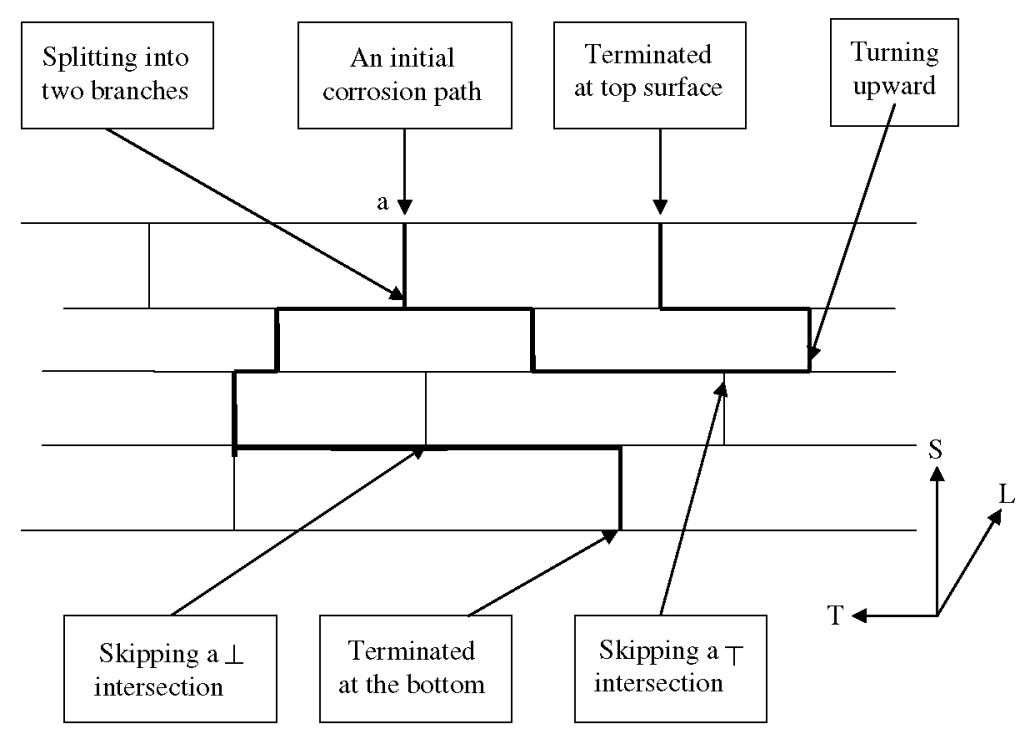

Fig. 2. The graph represents a corrosion path in a strip of aluminum alloy in the $\mathrm{S}$ direction, and the bold line stands for the growth of the IGC. For simplification, there is only one initial point "a" on the top surface.

Based on this discussion, we use a brick wall model similar to that proposed in Ruan et al. $(2004 a, b)$ to describe the influence of the grain size on the minimum IGC path length. Consider a strip of aluminum alloy with thickness $D$. We can estimate the number of vertical grain layers $k$ in the strip by $k=D / b$, where $b$ is the median of the estimated gamma distribution model for the grain size in the vertical (thickness) direction. Suppose there are a fixed number, $m$, of initial corrosion points on the surface of the alloy. For $i=1, \ldots, m$, let $W_{i, D}$ denote the distance that the $i$ th corrosion path travels to go through the thickness $D$ of the aluminum alloy. From Fig. 2 (a graphical representation of our brick wall model), the corrosion path starts from the top and travels along a vertical grain boundary until it reaches an intersection (arrives at the next layer). At this time it might split into two separate branches in opposite directions (in which case we treat them as two independent corrosion paths with a common previous path), or it might simply turn either left or right. In the latter case, it moves along the horizontal direction until it meets another intersection. There are two types of three-way intersections (" $T$ " and " $\perp$ "), so the corrosion path may have a chance to turn upward at the " $\perp$ " type intersection or downward at the "T" type intersection. If it does not turn upward or downward, it continues to propagate along the horizontal grain boundary, which means the corrosion path skips an intersection. If the 
corrosion path turns upward and arrives at the surface, we assume it is terminated because corrosion paths do not continue to travel on the top surface. Likewise, if the corrosion path turns downward and reaches the bottom, it is terminated. We assume that these are the only situations in which a corrosion path is terminated.

Let $p_{\text {up }}$ represent the probability that a corrosion path turns upward at a " $\perp$ " type intersection and let $p_{\text {sup }}$ represent the probability that a corrosion path skips a " $\perp$ " type intersection. Similarly, let $p_{\text {down }}$ denote the probability that a corrosion path turns downward at a "T" type intersection and let $p_{\text {sdown }}$ denote the probability that a corrosion path skips a "T" type intersection. Then, according to our previous discussion, we have

$$
p_{\text {up }}+p_{\text {sup }}=1
$$

and

$$
p_{\text {down }}+p_{\text {sdown }}=1 \text {. }
$$

In addition, let $p_{\text {split }}$ represent the probability that a corrosion path splits into two branches.

Suppose there are $m$ initial corrosion paths at the surface of the aluminum alloy. Let $u \geq 0$ be the number of additional paths resulting from splitting, and let $v \geq 0$ be the number of branches terminated at the top surface. Then, there are a total of $m+u-v$ corrosion paths traveling through the alloy from the top surface to the bottom. Let $W_{\min , D}$ denote the minimum IGC path length for thickness $D$. Then,

$$
W_{\min , D}=\min W_{i, D}, \quad i=1, \ldots, m+u-v .
$$

\subsection{Simulation}

Since the fastest IGC rate is the matter of concern, we study the minimum IGC length for each of the three directions (L, T and S) in our simulation. An IGC path growing in the S direction may turn in either the $\mathrm{L}$ or $\mathrm{T}$ direction, and turning in the $\mathrm{T}$ direction will result in a shorter path because the typical grain size in the $\mathrm{T}$ direction is shorter than that in the L direction. So, when we study a corrosion path in the $\mathrm{S}$ direction, as depicted in Fig. 2, we only care about turning in the $\mathrm{T}$ direction. Similarly, for an IGC path in either the $\mathrm{L}$ or $\mathrm{T}$ direction, the shorter path corresponds to turns in the $\mathrm{T}$ direction.

We use the programming language $\mathrm{R}$ to simulate the minimum IGC length in each of these three directions. For simplicity in our study, we assume that the probability of skipping an intersection is the same for both types ("T" and " $\perp$ ") intersections; that is, $p_{\text {sup }}=p_{\text {sdown }}=p_{\text {skip. }}$. Let $b_{j}, j=1, \ldots, k$, be the thickness of the $j$ th layer, which is generated from a gamma distribution with parameters obtained from Eqs. (1) and (2). Then, the thickness is $D=\sum_{j=1}^{k} b_{j}$. Let $X=1$ after a corrosion path has traveled through the first grain. If a corrosion path turns upward, then $X$ is reduced by one. If a corrosion path turns downward, then $X$ is increased by one. If $X=0$, it means that the corrosion path arrives at the top surface and is terminated. If $X=k$, the corrosion path reaches the bottom surface and the path is ended as well. Let $D_{i}(j)$ be the vertical distance that the $i$ th corrosion path travels along the width of the grain in the $j$ th layer. The first step in the simulation (Fig. 3) is a vertical step so that $D i(1)=b_{1}$. When a corrosion path reaches an intersection in the next layer, we need to assign $p_{\text {split }}$. Suppose $p_{\text {split }}=0.1$. Then a random number $z$ is generated from the uniform $(0,1)$ distribution. If $z<0.1$, then the path will split into two 
independent branches in opposite horizontal directions. If $z>0.1$, it will simply turn left or right, in which case we need to decide if the next intersection in the horizontal direction is " $T$ " or " $\perp$ ".

From Fig. 4, after the vertical step the corrosion path turns left along the boundary of the grains "a" and " $\mathrm{b}$ ". Under the discussed assumptions, we use a gamma distribution to model the grain size in each direction. So, $R_{b}$ can be represented by " $r G$ ", a random value generated from the estimated gamma. Moreover, since the vertical step could potentially arrive at any point along the boundary of grain "a", we let $R_{\mathrm{a}}=r \mathrm{U}(1) * r G$, where $r \mathrm{U}(1)$ is a random number generated from the uniform $(0,1)$ distribution and $r \mathrm{U}(1)$ and $r G$ are taken to be independent. Let $L_{\mathrm{up}}=R_{\mathrm{b}}$ and $L_{\mathrm{d}}=R_{\mathrm{a}}$. If $L_{\mathrm{up}}>L_{\mathrm{d}}$, then the next intersection is "T" (which is the case in Fig. 4). If $L_{\mathrm{up}}<L_{\mathrm{d}}$ then the next intersection is " $\perp$ ". (Since the four-way intersection is rare, we assume $\mathrm{P}\left(L_{\mathrm{up}}=L_{\mathrm{d}}\right)$ $=0$.) Suppose the next intersection is "T". Then we need to assign $p_{\text {sdown }}$. Supposing $p_{\text {sdown }}=0.2$, for example, a random number $z$ is generated from the uniform $(0,1)$ distribution. If $z \geq 0.2$, the corrosion path will turn downward. If $z<0.2$, it will skip the intersection and $L_{d}=L_{d}+r G$, which is $R_{\mathrm{a}}+R_{\mathrm{c}}$. Then we can compare the "new" $L_{d}$ with $L_{\mathrm{up}}$ to simulate the form of the next intersection (in Fig. 4, $R_{\mathrm{a}}+R_{\mathrm{c}}>R_{\mathrm{b}}$, so the next intersection is " $\perp$ "). When the next intersection is

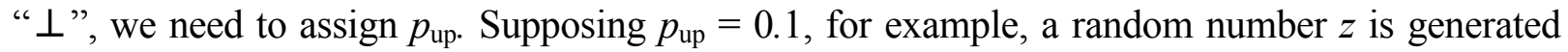
from the uniform $(0,1)$ distribution. If $z \leq 0.1$, the corrosion path will turn upward. If $z>0.1$, it will skip the intersection and $L_{\text {up }}=L_{\text {up }}+r G=R_{\mathrm{b}}+R_{\mathrm{d}}$. Then we can compare the "new" $L_{\text {up }}$ with $L_{\mathrm{d}}$ to figure out the form of the next intersection. (In Fig. 4, $R_{\mathrm{a}}+R_{\mathrm{c}}<R_{\mathrm{b}}+R_{\mathrm{d}}$, so the next intersection is "T".) We continue this process until the branch is terminated at the top or bottom surface. This procedure is repeated until all the branches are simulated. The shortest one (among all the paths that go completely through the alloy from the top surface to the bottom surface) is then the minimum IGC path for that simulation. 


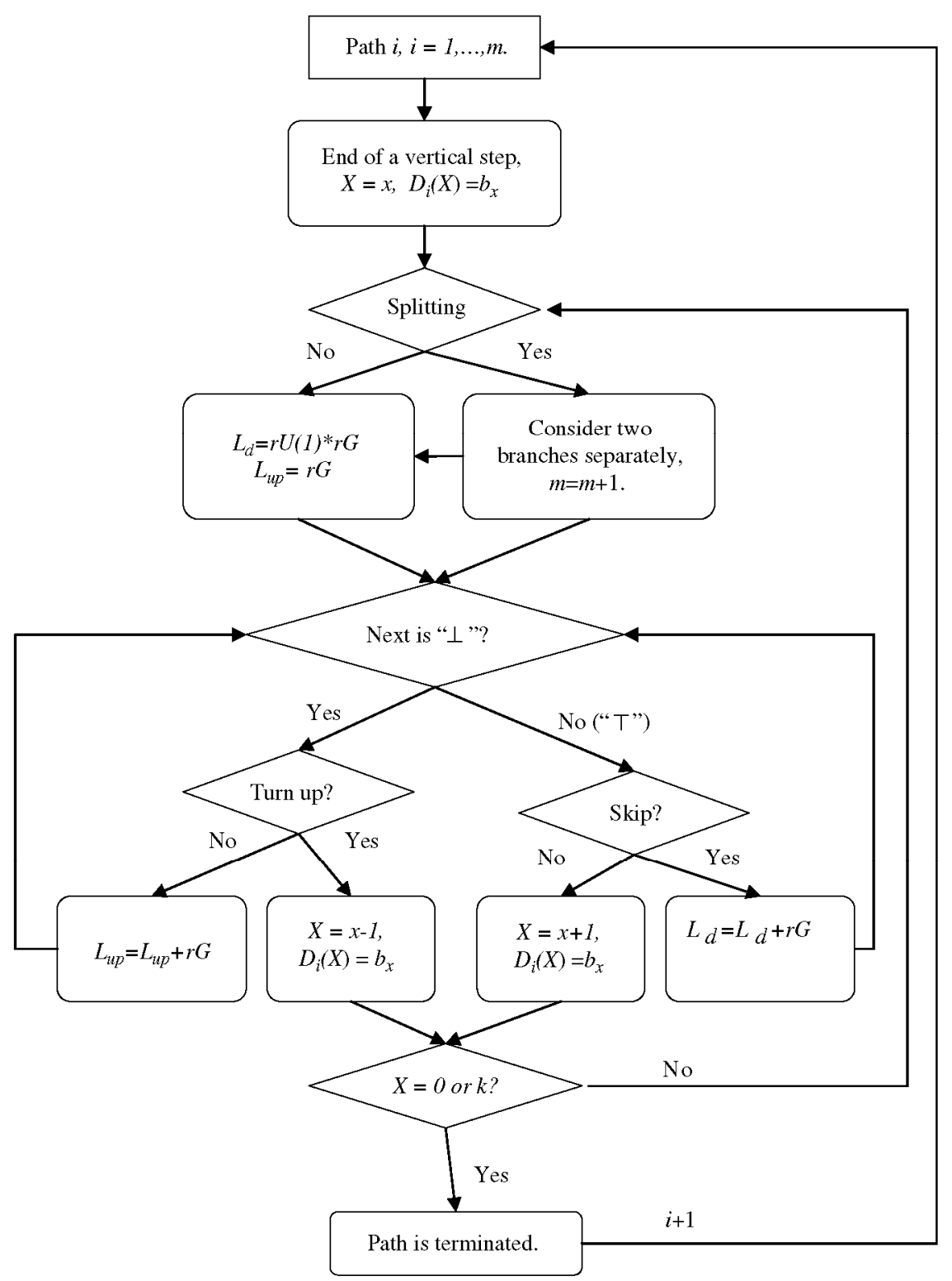

Fig. 3. A flow-chart for the computer simulation.

\section{Comparison of experimental data with the simulation results}

From the study by Huang (2004), grain sizes of aluminum alloys Equi-axed AA7178-T6 and Wingskin AA7178-T6 are given in Table 1.We see that the grain size of Equi-axed AA7178-T6 is similar in all three directions, while Wingskin AA7178-T6 has elongated grain size in the L and T directions, but shorter grain size in the $\mathrm{S}$ direction. Thus based on the previous discussion, the corrosion in Equi-axed AA7178-T6 is isotropic, while the corrosion in Wingskin AA7178-T6 is anisotropic with the fastest nominal IGC growth rate in the longitudinal (L) or long transverse (T) direction and the slowest rate in the short transverse direction (S), since the IGC path will be straight along either the $\mathrm{L}$ or $\mathrm{T}$ directions and circuitous in the $\mathrm{S}$ direction.

From the previous discussion, we use gamma distributions to model grain sizes for the three directions in both alloys. Parameters for these gamma distributions obtained using Eqs. (1) and (2) are given in Table 2. 


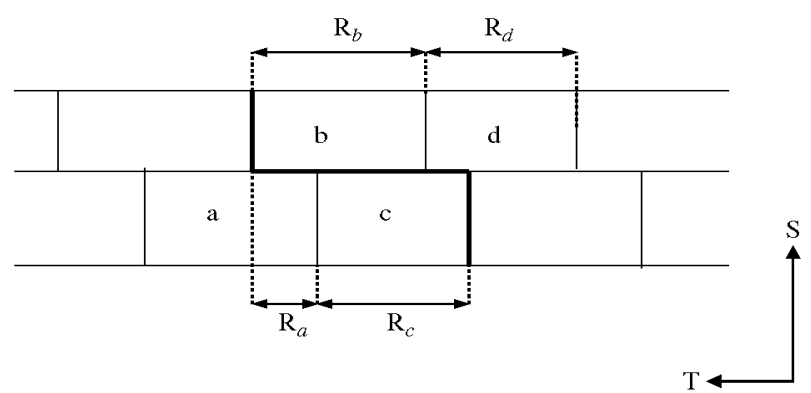

Fig. 4. The bold line represents a corrosion path, a, b, c and $\mathrm{d}$ stand for grains and $\mathrm{R}$ represents the random number generated by the simulation.

Table 1

Grain dimensions of Equiaxed AA7178-T6 and Wingskin AA7178-T6

\begin{tabular}{llc}
\hline Orientation & Equi-axed AA7178-T6 $(\mu \mathrm{m})$ & Wingskin AA7178-T6 $(\mu \mathrm{m})$ \\
\hline $\mathrm{L}$ & $183.9 \pm 68.9$ & $1040 \pm 692$ \\
$\mathrm{~T}$ & $189.2 \pm 64.2$ & $511 \pm 261$ \\
$\mathrm{~S}$ & $174.6 \pm 56.0$ & $39 \pm 17$ \\
\hline
\end{tabular}

Table 2

Gamma models for grain size distributions for each direction of Equiaxed AA7178-T6 and Wingskin AA7178-T6

\begin{tabular}{lll}
\hline Orientation & Equi-axed AA7178-T6 & Wingskin AA7178-T6 \\
\hline $\mathrm{L}$ & $\operatorname{gamma}(7.13,25.81)$ & $\operatorname{gamma}(2.26,460.45)$ \\
$\mathrm{T}$ & $\operatorname{gamma}(8.69,21.78)$ & $\operatorname{gamma}(3.83,133.3)$ \\
$\mathrm{S}$ & $\operatorname{gamma}(9.72,17.96)$ & $\operatorname{gamma}(5.26,7.41)$ \\
\hline
\end{tabular}

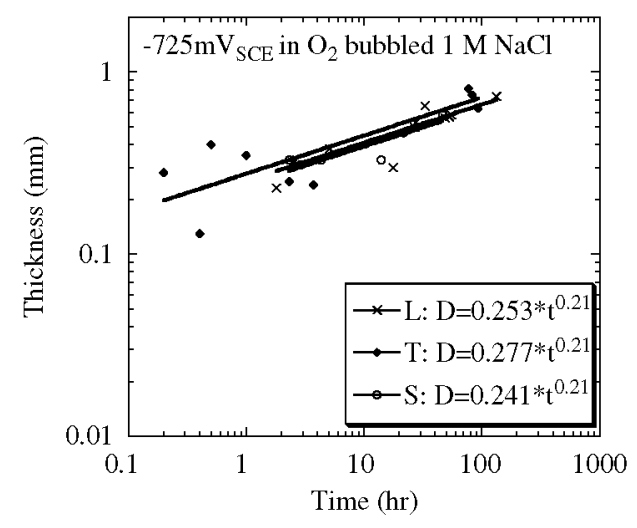

Fig. 5. Localized corrosion kinetics of Equi-axed AA7178-T6 by fitting data to the equation $D=A \times t^{n}$, with $n=$ 0.21 .

On the basis of foil penetration experiments for Equi-axed AA7178-T6 and Wingskin AA7178-T6, Huang and Frankel (2004) observed the thickness of the foil and the shortest times for the corrosion to penetrate that thickness in each direction for the two materials. They found that an equation of the form $D=A \times t^{n}$ can be used to fit the experimental data, where $D$ denotes thickness, $t$ is time, $n$ is a constant that depends on the particular alloy and $A$ is a constant that depends on both the alloy and the direction of the IGC within the alloy. 
From Figs. 5 and 6, we find that $A$ is proportional to the corrosion rate in the given direction, although the goodness of the fit for the relationship varies a bit across the different directions within a given alloy. The larger $A$ is, the faster the corrosion rate will be in that direction. In our statistical model, we consider the local intergranular growth speed to be the same in every direction for each material, so that a faster corrosion rate corresponds to a shorter minimum IGC path length. Therefore, since $A$ is proportional to the corrosion rate, it is inversely related to the minimum IGC path length for a given thickness. For Equi-axed AA7178-T6, the ratio of $A^{-1}$ in the $\mathrm{L}, \mathrm{T}$ and $\mathrm{S}$ directions is 1:0.91:1.05, while for Wingskin AA7178-T6 the ratio of $A^{-1}$ in the L, $\mathrm{T}$ and $\mathrm{S}$ directions is 1:1.03:4.01.

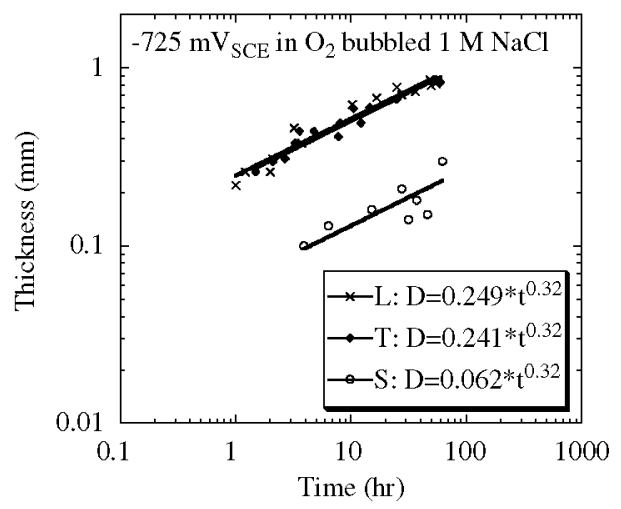

Fig. 6. Localized corrosion kinetics of Wingskin AA7178-T6 by fitting data to the equation $D=A \times t^{n}$, with $n=$ 0.32 .

Table 3

Simulation results for some combinations of the probabilities $\left(p_{\text {up }}, p_{\text {skip }}, p_{\text {split }}\right)$

\begin{tabular}{|c|c|c|c|c|c|}
\hline Observation & $p_{\text {up }}$ & $p_{\text {skip }}$ & $p_{\text {split }}$ & $\begin{array}{l}\text { Ratio of minimum IGC } \\
\text { path lengths for Equi-axed } \\
\text { AA7178-T6-L:T:S }\end{array}$ & $\begin{array}{l}\text { Ratio of minimum IGC } \\
\text { path lengths for Wingskin } \\
\text { AA7178-T6-L:T:S }\end{array}$ \\
\hline 1 & 0.1 & 0.5 & 0.05 & $1: 1: 1$ & $1: 1: 5.9$ \\
\hline 2 & 0.1 & 0.3 & 0.05 & $1: 1: 1$ & $1: 1: 4.3$ \\
\hline 3 & 0.1 & 0.2 & 0.05 & $1: 1: 1$ & $1: 1: 4.1$ \\
\hline 4 & 0.1 & 0.1 & 0.05 & $1: 1: 1$ & $1: 1: 3.5$ \\
\hline 5 & 0.1 & 0.15 & 0.05 & $1: 1: 1$ & $1: 1: 3.8$ \\
\hline 6 & 0.1 & 0.17 & 0.05 & $1: 1: 1$ & $1: 1: 4.0$ \\
\hline
\end{tabular}

Table 4

Simulation results for additional combinations of the probabilities ( $\left.p_{\text {up }}, p_{\text {skip }}, p_{\text {split }}\right)$

\begin{tabular}{llllll}
\hline Observation & $p_{\text {up }}$ & $p_{\text {skip }}$ & $p_{\text {split }}$ & $\begin{array}{l}\text { Ratio of minimum IGC } \\
\text { path lengths for Equi-axed } \\
\text { AA7178-T6-L:T:S }\end{array}$ & $\begin{array}{l}\text { Ratio of minimum IGC } \\
\text { path lengths for Wingskin } \\
\text { AA7178-T6-L:T:S }\end{array}$ \\
\hline 1 & 0.2 & 0.2 & 0.05 & $1: 1: 1$ & $1: 1: 4.2$ \\
2 & 0.2 & 0.1 & 0.05 & $1: 1: 1$ & $1: 1: 3.7$ \\
3 & 0.2 & 0.15 & 0.05 & $1: 1: 1$ & $1: 1: 3.8$ \\
4 & 0.2 & 0.18 & 0.05 & $1: 1: 1$ & $1: 1: 4.1$ \\
5 & 0.2 & 0.16 & 0.05 & $1: 1: 1$ & $1: 1: 4.0$ \\
\hline
\end{tabular}

Using the simulation algorithm described in Section 2.2, we investigated the effect of $p_{\text {up }}, p_{\text {skip, }}$ and $p_{\text {split }}$ on the minimum IGC path length in the L, T and $\mathrm{S}$ directions for both Equi-axed AA7178-T6 and Wingskin AA7178-T6. Results are presented in Table 3 for some combinations of ( $\left.p_{\text {up }}, p_{\text {skip }}, p_{\text {split }}\right)$.

From examining the experimental data, it appears that both $p_{\text {up }}$ and $p_{\text {split }}$ are rather small. Thus 
in our simulations we first set $p_{\text {up }}=0.1$ and $p_{\text {split }}=0.05$ and then adjusted $p_{\text {skip }}$ to provide good agreement with the experimental ratios L:T:S, namely, 1:0.91:1.05 for Equi-axed AA7178-T6 and 1:1.03:4.01 for Wingskin AA7178-T6. Observation 6 in Table 3 provides a good fit to the observed ratios for both alloys, which tells us that the combination of probabilities ( $p_{\text {up }}, p_{\text {skip, }}$ $\left.p_{\text {split }}\right)=(0.1,0.17,0.05)$ is tenable. But, of course, this combination is not unique. There are other ( $\left.p_{\text {up }}, p_{\text {skip }}, p_{\text {split }}\right)$ combinations that will also provide good fits to the observed L:T:S ratios. For example, we see in Table 4 that $\left(p_{\text {up }}, p_{\text {skip }}, p_{\text {split }}\right)=(0.2,0.16,0.05)$ also provides a good fit to the experimental data.

\section{Conclusions}

In this paper, we study a more realistic modified brick wall model than the one proposed by Ruan et al. (2004a,b). In their model, when a corrosion path meets an intersection, it was assumed to be a four-way intersection. In most alloys, however, three-way intersections are more common. In our model, intersections are divided into two three-way types ("T" and " $\perp$ "), and in our simulation we use a random mechanism to decide whether a given intersection is " $T$ " or " $\perp$ ". Moreover, we discuss the corrosion growth kinetics for all the three directions rather than only consider the $\mathrm{S}$ direction.

As we pointed out previously, there is no unique combination of probabilities ( $p_{\text {up }}, p_{\text {skip }}, p_{\text {split }}$ ) that provides an optimal fit to the experimental data. Nevertheless, the information in Tables 3 and 4 gives us some insight about possible combinations of the probabilities ( $\left.p_{\text {up }}, p_{\text {skip }}, p_{\text {split }}\right)$ that provide reasonable fits to the data. For example, observation 6 in both Tables 3 and 4 provide good fits to the data.

Our simulation results suggest that $p_{\text {up }}$ does not have an obvious effect on the ratio when both $p_{\text {skip }}$ and $p_{\text {split }}$ are small. On the other hand, $p_{\text {skip }}$ has a clear effect on the ratio when $p_{\text {split }}$ is small. This can be explained from the simulation algorithm described in Section 2.2. In Fig. 4, we know that after the vertical step, we will compare $R_{a}$ with $R_{\mathrm{b}}$ to decide if the first intersection in the horizontal direction is " $T$ " or " $\perp$ ". Note that $R_{\mathrm{b}}$ can be represented by " $r G$ ", a random value generated from the appropriate estimated gamma distribution, while $R_{\mathrm{a}}=r \mathrm{U}(1) * r G$, where $r \mathrm{U}(1)$ is a random number generated from the uniform $(0,1)$ distribution. Thus, $R b>R_{\mathrm{a}}$ in most cases, which means that the first intersection is usually "T". Thus, $p_{\text {up }}$ will not have much effect on the ratio when $p_{\text {skip }}$ is small, since the corrosion path will not often have an opportunity to meet a " $\perp$ " intersection and turn upward.

We expect that our model will also be appropriate for non-aluminum alloys for which the corrosion is primarily focused along the grain boundaries. In our study, however, we found other materials that have IGC corrosion properties that are not approximated well by our model. For example, IGC corrosion in the aluminum alloy Rolled AA7075-T6 can also attack the grain interiors while propagating along the grain boundaries. This makes the corrosion rate much slower in Rolled AA7075-T6 than in other materials with similar grain sizes. In materials such as Rolled AA7075-T6, we will need to modify our model to accommodate interior as well as boundary IGC corrosion in the grains.

\section{References}

Guillaumin, V., Mankowski, G., 1999. Localized corrosion of 2024 T351 aluminum alloy in chloride media. Corrosion Sci. 41, 421-438.

Huang, T., Frankel G.S., 2004. Intergranular corrosion morphology and growth kinetics 
in AA7075 and AA7178. Proceedings of the 206th Meeting of The Electrochemical Society, Honolulu, Hawaii.

Liu, X., Frankel, G.S., Zoofan, B., Rokhlin, S.I., 2004. Effect of applied tensile stress on intergranular corrosion of AA2024-T3. Corrosion Sci. 46, 405-425.

Robinson, M.J., Jackson, N.C., 1999. The influence of grain structure and intergranular corrosion rate on exfoliation and stress corrosion cracking of high strength $\mathrm{Al}-\mathrm{Cu}-\mathrm{Mg}$ alloys. Corrosion Sci. 41, 1013-1028.

Ruan, S., Wolfe, D.A., Zhang, W., Frankel, G.S., 2004a. Statistical modeling of minimum intergranular corrosion path length in high strength aluminum alloy. Technometrics 46, 69-75.

Ruan, S., Wolfe, D.A., Frankel, G.S., 2004b. Statistical modeling and computer simulation of intergranular corrosion growth inAA2024-T3 aluminum alloy. J. Statist. Plann. Inference 126, 553-568. 